\title{
The Association between Socioeconomic Status and Adherence to Health Check- up in Korean Adults, Based on the 2010-2012 Korean National Health and Nutrition Examination Survey
}

\author{
Hyun-Young Shin ${ }^{1,2}$, Hee-Taik Kang ${ }^{3, *}$, Jae Woo Lee, ${ }^{4, *}$, Hyoung-Ji Lim ${ }^{4}$ \\ 'Department of Family Medicine, Myongji Hospital, Goyang, Korea \\ ${ }^{2}$ Department of Epidemiology and Health Promotion and Institute for Health Promotion, Yonsei University Graduate School of Public Health, Seoul, \\ Korea \\ ${ }^{3}$ Department of Family Medicine, Chungbuk National University College of Medicine, Cheongju, Korea \\ ${ }^{4}$ Department of Family Medicine, Chungbuk National University Hospital, Cheongju, Korea
}

\begin{abstract}
Background: We investigated the association between socioeconomic status and adherence to health check-ups in a Korean population aged 40 years or older.

Methods: This cross-sectional study included 12,311 participants who participated in the 2010-2012 Korean National Health and Nutrition Examination Survey. Self-reported questionnaires were used to assess each participant's socioeconomic status (household income, occupation, and education) and adherence to health check-ups. Results: Men with a higher income (highest vs. lowest: odds ratio [OR], 1.799; 95\% confidence interval [CI], 1.2962.497 ) and men with a higher education level ( $\geq 12$ vs. $<6$ years: OR, 1.488; 95\% CI, 1.078-2.054) and office workers compared with manual workers (men: OR, 1.431; 95\% CI, 1.077-1.902; women: OR, 1.783; 95\% CI, 1.256-2.532) appeared to undergo more health check-ups. In particular, men and women with a higher income and education appeared more likely to undergo opportunistic health check-ups (men: highest vs. lowest income: OR, 2.380; 95\% CI, 1.218-4.653; $\geq 12$ vs. $<6$ years education: OR, 2.121; 95\% CI, 1.142-3.936; women: highest vs. lowest income: OR, 4.042; 95\% CI, 2.239-7.297; $\geq 12$ vs. <6 years education: OR, 2.475; 95\% CI, 1.283-4.775).

Conclusion: A higher socioeconomic status was associated with a higher rate of participation in health check-ups. More efforts are needed to identify the factors associated with disparity in adherence to health check-ups.
\end{abstract}

Keywords: Education; Health; Income; Health Promotion; Public Health

Received: September 22, 2016, Revised: April 27, 2017, Accepted: May 7, 2017

${ }^{*}$ Corresponding Author: Hee-Taik Kang https://orcid.org/0000-0001-8048-6247

Tel: +82-43-269-6301, Fax: +82-43-269-6675, E-mail: kanght@chungbuk.ac.kr

${ }^{*}$ Corresponding Author: Jae Woo Lee https://orcid.org/0000-0003-1784-6494

Tel: +82-43-269-6301, Fax: +82-43-269-6675, E-mail: shrimp0611@gmail.com

*These co-corresponding authors equally contributed to this work. 


\section{INTRODUCTION}

With increases in human life expectancy, health policies have prioritized health promotion and disease prevention for noncommunicable diseases such as cardiovascular diseases, metabolic disorders, and cancer. ${ }^{1)}$ Health policies in South Korea have progressed with respect to the prevention of noncommunicable diseases, and the national health insurance system, which has covered all Koreans since 1989, provides health check-ups for secondary prevention of disease. ${ }^{2)}$

Socioeconomic status is associated with health-related behaviors and disease outcomes. ${ }^{3-6)}$ In particular, it appears that socioeconomic status affects lifestyle factors such as alcohol consumption, cigarette smoking, physical exercise, and dietary patterns, which are closely related to noncommunicable diseases. ${ }^{3-5)}$ Furthermore, individuals with a low socioeconomic status are more likely to be surrounded by an unhealthy environment, and they have a higher risk for health problems such as obesity, cardiovascular disease, and cancer, resulting in poor health outcomes. ${ }^{5,7,8)}$ Furthermore, socioeconomic deprivation makes it difficult for those individuals to take advantage of health check-up programs because of reduced access to healthcare hospitals. ${ }^{9-11)}$

Understanding the linkage between socioeconomic status and adherence to health check-ups would be helpful for directing health policy and distributing limited public health resources fairly and efficiently. The aim of this study was to investigate the association between socioeconomic status (household income level, occupation, and education) and adherence to health check-ups after adjusting for confounding variables in Korean individuals aged 40 years and older. In addition, we examined the association between socioeconomic status and participation in opportunistic and national health check-ups, separately.

\section{METHODS}

\section{Study Population}

The Korean Ministry of Health and Welfare periodically conducts the Korean National Health and Nutrition Examination Survey (KNHANES), which is a nationwide survey representing the entire Korean population. This survey includes comprehensive information on health status, health behavior, socioeconomic demographics, and laboratory tests. As the sampling units, houses were selected based on a stratified, multistage, probability-sampling design according to age, sex, and geographic area. Participants were requested to complete four types of evaluation, including a health interview, health examination, and questionnaires on health behavior and nutrition. Trained interviewers performed the health interview using face-to-face interviews at participants' homes. Citizens could refuse to participate in this survey according to the National Health Enhancement Act. Written informed consent was provided by participants when they agreed to participate. For further research, the Korea Centers for Disease Control and Prevention obtained participants' consent to use blood samples obtained during the health interview survey. Trained staff followed standardized steps to conduct the unified procedures. Body weight and height were measured to the nearest $0.1 \mathrm{~kg}$ and $0.1 \mathrm{~cm}$, respectively, while participants were wearing light indoor clothes without shoes. Body mass index was calculated as the ratio of weight in kilograms to squared height in meters $\left(\mathrm{kg} / \mathrm{m}^{2}\right)$. Detailed information about KNHANES has been reported in a previous study. ${ }^{12)}$

This study was based on data from 25,532 adults in 192 national districts obtained from the 2010-2012 KNHANES V after merging each year's data. Among them, 13,661 adults aged over 40 years old were included in this study. Although it might be different by inspection item in health check-ups, the recommended age for most examinations is over 40 years old. Moreover, opportunistic comprehensive health check-ups are mainly performed in middle-aged and elderly people. Individuals who did not have data regarding socioeconomic variables such as household income, occupation, education, and marital status and those who did not have information regarding the adherence to health check-ups were excluded. After these exclusions, 12,311 participants (5,288 men and 7,023 women) were included in the final analysis (Supplementary Figure 1). This study was approved by the Institutional Review Board of Chungbuk National University Hospital (IRB no., CBNUH 2017-03-012) and written informed consents were obtained.

\section{Definitions of Socioeconomic and Health Check-up Variables}

Information on socioeconomic status and health-related behaviors was obtained from data gathered from the self-reported questionnaire administered during the interview from the relevant survey period. Men who ingested more than 7 alcoholic drinks and women who ingested more than 5 alcoholic drinks at a frequency of more than twice a week were categorized as high-risk drinkers. Individuals who smoked cigarettes during the relevant survey period were categorized as current smokers. Vigorous physical activity at least 3 days per week or moderate physical activity, including walking, at least 5 days per week was defined as regular exercise. ${ }^{13)}$ Daily calorie intake was monitored by 24-hour food recall and analyzed using a computer-aided nutritional analysis program (CAN-Pro ver. 3.0 software; Korean Nutrition Society, Seoul, Korea). ${ }^{14)}$ Participants answered an open-ended question on income: "What is your average monthly income including salaries, property income, pension, government subsidies, and allowance?" Monthly average household income was calculated by dividing total monthly house income by the number of family members. The Ministry of Health and Welfare classifies individuals into four groups (lowest, middle-lowest, middle-highest, and highest) according to monthly household income. Residential area was divided into metropolitan and other. Metropolitan residents are individuals living in in the seven largest cities (Seoul, Busan, Daegu, Incheon, Gwangju, Daejeon, and Ulsan) in Korea. Insurance type was categorized as national health insurance (regionally insured and workplace insured) or medical aid. Employment was classified into manual work (clerks; service and sales workers; skilled agricultural, forestry, and fishery workers; persons who operate or assemble crafts, equipment, or machines; and 
elementary workers), office work (general managers, government administrators, professionals, and simple office workers), and other (unemployed persons, housekeepers, and students). Educational status was classified according to duration into $<6,6-8,9-11$, and $\geq 12$ years. Marital status was classified into three groups: married and not separated (i.e., people who are married and live together), single (i.e., people who have not married, people who have been married but are now separated, people who were married but whose spouses passed away, and people who are divorced), and no response.

Participants answered questions regarding adherence to health check-ups, such as, "Have you undergone a health check-up within the last 2 years?" Participants who answered "yes" were further questioned about the type of health check-up they underwent. Health check-ups were additionally categorized into opportunistic and national health check-ups. Opportunistic health check-ups are screening tests for which participants pay the hospital costs for check-ups performed in private healthcare sectors such as private general hospitals, clinics, and health check-up centers, as well as in public healthcare sectors such as the Korean National Health Insurance Corporation. National health check-ups are screening tests including industry special health examinations, general health check-ups provided by the Korean National Health Insurance Corporation, and health check-ups provided by other government organizations. ${ }^{2)}$ Participants who answered "yes" to "Have you ever been diagnosed with any kind of cancer or malignancy in your life?" were classified as having a cancer diagnosis. Those who answered "no" were the control group. We also asked the question, "Have you ever been diagnosed with hypertension, dyslipidemia, diabetes, and cardio- or cerebrovascular diseases in your life?" Respondents who answered the question "yes" were classified as having a chronic disease, and those who answered "no" were the control group. Unanswered questions were treated as missing values in our data.

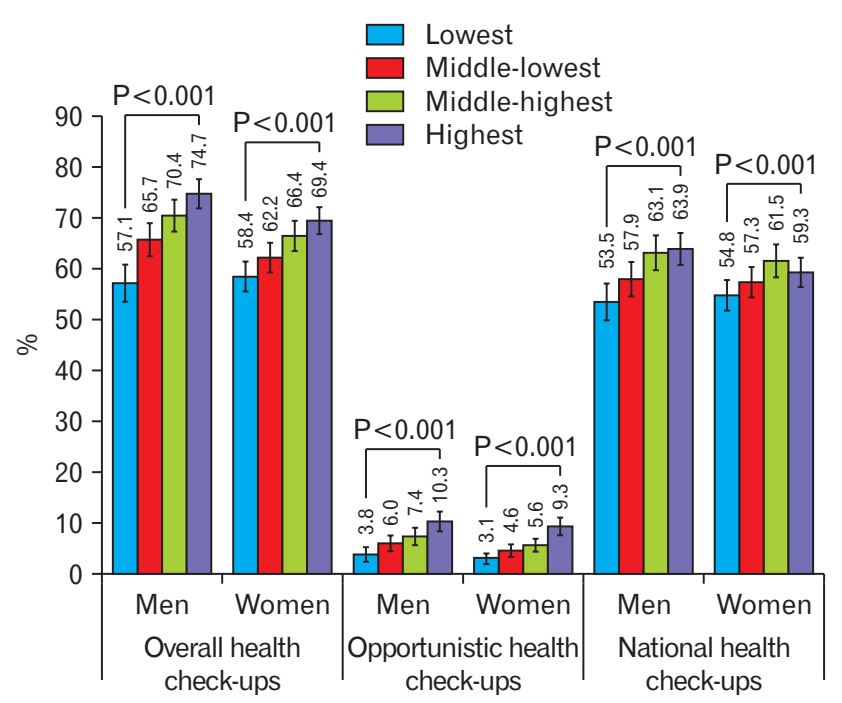

Figure 1. Percentage of adherence to health check-ups according to monthly household income, with each $\mathrm{P}$ trend $<0.001$.

\section{Statistical Analysis}

To represent the general Korean population with minimal bias, sampling weights were applied to account for the complex sampling. All data for continuous variables are presented as means and standard errors (SEs). Data for categorical variables are presented as percentages and SEs. To compare percentages for health check-ups within 2 years according to socioeconomic status, $\chi^{2}$ tests were used. $\mathrm{P}$ trend for household income status (Figure 1 ) and $\chi^{2}$ for occupational and educational status (Figures 2, 3) were calculated by overall, opportunistic, and national check-ups in men and women. Odds ratios (ORs) and 95\% confidence intervals (CIs) for health check-up performance within 2 years were calculated using weighted multivariate logistic regres-

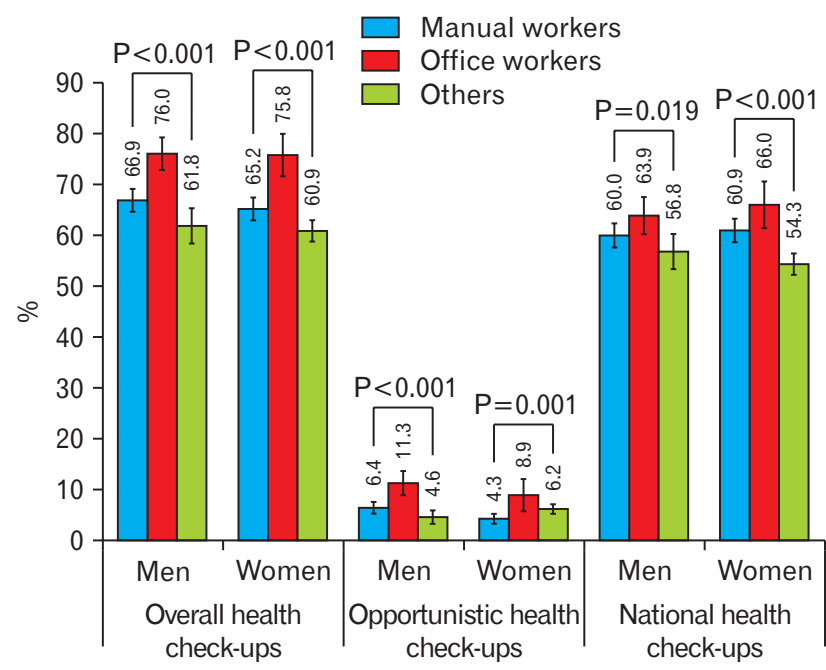

Figure 2. Percentage of adherence to health check-ups according to occupational status. P-values were calculated from $\chi^{2}$ tests comparing differences between groups.

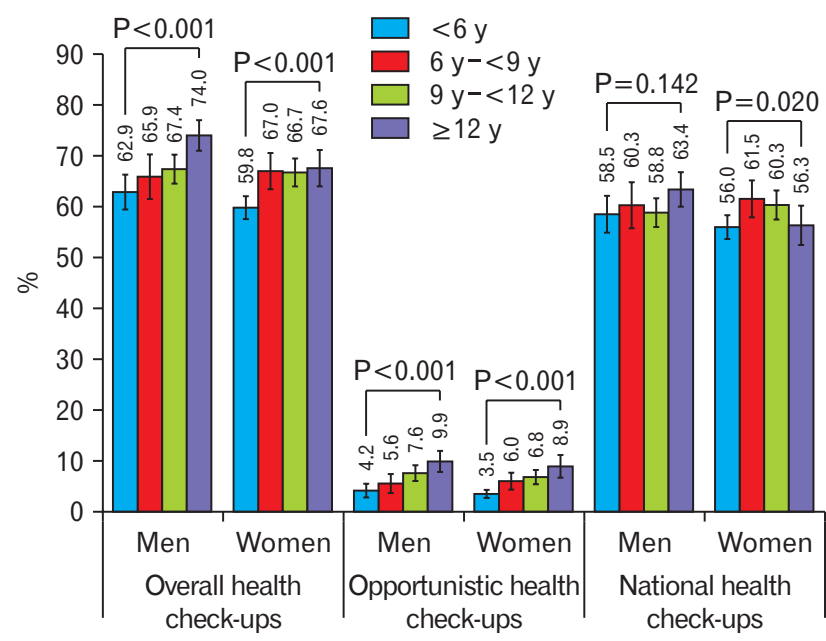

Figure 3. Percentage of adherence to health check-ups according to educational status. P-values were calculated from $\chi^{2}$ tests comparing differences between groups. 
sion analyses after adjusting for age, average monthly household income, residence, type of health insurance, occupation, education, and marital status (model 1). Next, we performed multivariate logistic regression analysis and adjusted for body mass index, daily calorie intake, smoking, alcohol use, and physical activity in addition to the variables in model 1 (model 2). Finally, the factors for chronic disease and cancer diagnosis were added to the adjusted variables of model 2 (model 3). SAS ver. 9.2 (SAS Institute Inc., Cary, NC, USA) was used to

Table 1. Characteristics of the study population

\begin{tabular}{|c|c|c|}
\hline Characteristic & Men & Women \\
\hline No. of participants (unweighted) & 5,288 & 7,023 \\
\hline Age (y) & $54.8 \pm 0.2$ & $56.6 \pm 0.2$ \\
\hline Body mass index $\left(\mathrm{kg} / \mathrm{m}^{2}\right)$ & $24.1 \pm 0.1$ & $24.1 \pm 0.1$ \\
\hline High-risk drinker (\%) & $26.4 \pm 0.9$ & $5.0 \pm 0.5$ \\
\hline Current smoker (\%) & $41.5 \pm 0.9$ & $5.0 \pm 0.4$ \\
\hline Daily calorie intake (kcal) & $2,355 \pm 19$ & $1,649 \pm 12$ \\
\hline Regular physical activity (\%) & $52.7 \pm 0.9$ & $48.5 \pm 0.8$ \\
\hline Household income ( $10^{4}$ Korean won/mo) & $459.6 \pm 17.8$ & $399.6 \pm 14.2$ \\
\hline Metropolitan residents (\%) & $44.2 \pm 1.3$ & $45.1 \pm 1.2$ \\
\hline \multicolumn{3}{|l|}{ Health insurance } \\
\hline National health insurance (\%) & $96.8 \pm 0.3$ & $95.6 \pm 0.3$ \\
\hline Medical aid (\%) & $2.8 \pm 0.3$ & $3.8 \pm 0.3$ \\
\hline \multicolumn{3}{|l|}{ Occupation (\%) } \\
\hline Manual worker & $55.6 \pm 1.0$ & $41.1 \pm 0.9$ \\
\hline Office worker & $24.1 \pm 0.9$ & $9.6 \pm 0.5$ \\
\hline Other & $20.2 \pm 0.7$ & $49.3 \pm 0.8$ \\
\hline \multicolumn{3}{|l|}{ Education duration (\%) } \\
\hline$<6 y$ & $20.7 \pm 0.8$ & $40.7 \pm 0.9$ \\
\hline $6-8 y$ & $15.7 \pm 0.7$ & $15.2 \pm 0.6$ \\
\hline $9-11$ y & $35.4 \pm 0.9$ & $29.9 \pm 0.8$ \\
\hline$\geq 12 y$ & $28.1 \pm 1.0$ & $14.2 \pm 0.7$ \\
\hline \multicolumn{3}{|l|}{ Marital status (\%) } \\
\hline Single & $6.6 \pm 0.4$ & $23.4 \pm 0.7$ \\
\hline Married and not separated & $89.8 \pm 0.6$ & $75.4 \pm 0.7$ \\
\hline No response & $3.6 \pm 0.4$ & $1.1 \pm 0.2$ \\
\hline \multicolumn{3}{|c|}{ Adherence to health check-ups within 2 years (\%) } \\
\hline Overall health check-ups & $68.1 \pm 0.9$ & $64.1 \pm 0.8$ \\
\hline Opportunistic check-ups & $7.2 \pm 0.5$ & $5.6 \pm 0.3$ \\
\hline National check-ups & $60.3 \pm 0.9$ & $58.2 \pm 0.8$ \\
\hline Chronic disease (\%) & $31.9 \pm 0.8$ & $34.4 \pm 0.8$ \\
\hline Cancer diagnosis (\%) & $3.1 \pm 0.3$ & $5.0 \pm 0.3$ \\
\hline
\end{tabular}

Values are presented as mean \pm standard error or $\% \pm$ standard error. High-risk drinkers were defined as men who ingest more than 7 cups of alcohol and women who ingest more than 5 cups of alcohol per day at a frequency of more than twice a week. Regular physical activity is defined as vigorous-intensity activity $\geq 3 \mathrm{~d} /$ wk or moderate-intensity activity including walking $\geq 5 \mathrm{~d} / \mathrm{wk}$. Household income: total monthly house income/no. of family members. Chronic diseases include hypertension, dyslipidemia, diabetes, and cardio-/cerebrovascular disease. Metropolitan residents included those living in the 7 largest cities (Seoul, Busan, Daegu, Incheon, Gwangju, Daejeon, and Ulsan) in Korea. Insurance type was categorized as national health insurance (regionally insured and workplace insured) and medical aid. Occupation was categorized as office workers (general managers, government administrators, professionals, and other office workers), manual workers (clerks; service and sales workers; skilled agricultural, forestry, and fishery workers; persons who operate or assemble craft, equipment, or machines; and elementary workers), and other (unemployed persons, housekeepers, and students). Marital status was categorized as married and not separated, single (not married, separated, divorced, or widowed), and no response. conduct statistical analyses. All statistical tests were two-tailed, and statistical significance was determined at $\mathrm{P}<0.05$.

\section{RESULTS}

Subject characteristics are summarized in Table 1 . The mean age was 54.8 years for men and 56.6 years for women. Rates of overall, opportunistic, and national health check-ups within 2 years were $68.1 \%$, $7.2 \%$, and $60.3 \%$ in men and $64.1 \%, 5.6 \%$, and $58.2 \%$ in women, respectively.

Figures 1-3 show the percentage of participants who underwent health check-ups within the previous 2 years according to socioeconomic status. The highest household income groups showed the highest percentage of adherence to overall health check-ups in both sexes (74.7\% in men and $69.4 \%$ in women, $\mathrm{P}$ trend $<0.001$ in both sexes). Furthermore, these groups underwent the highest percentage of opportunistic and national health check-ups in men (10.3\% and $63.9 \%, \mathrm{P}$ trend $<0.001$ in both sexes) and the highest percentage of opportunistic health check-ups in women $(9.3 \%$, P trend $<0.001)$ (Figure 1$)$. Office workers underwent the highest rates of overall, opportunistic and national health check-ups in men and women $(76.0 \%, 11.3 \%$, and $63.9 \%$ in men and $75.8 \%, 8.9 \%$, and $66.0 \%$ in women, respectively; $\mathrm{P}<0.001$, $\mathrm{P}<0.001$, and $\mathrm{P}=0.019$ in men; $\mathrm{P}<0.001, \mathrm{P}=0.001$, and $\mathrm{P}<0.001$ in women) (Figure 2). The most educated individuals underwent the highest rates of overall, opportunistic, and national health check-ups in men (74.0\%, 9.9\%, and 63.4\%, respectively; $\mathrm{P}<0.001, \mathrm{P}<0.001$, and $\mathrm{P}=0.142$ ); however, in women, only overall and opportunistic health check-ups were performed most frequently in the most educated group (67.6\% and $8.9 \%$; $\mathrm{P}<0.001$ for both) (Figure 3 ).

In Table 2, we present the multivariate logistic regression results for

Table 2. Adherence to health check-ups according to socioeconomic status

\begin{tabular}{|c|c|c|}
\hline \multirow{2}{*}{ Variable } & \multicolumn{2}{|c|}{ OR (95\% Cl) } \\
\hline & Men & Women \\
\hline \multicolumn{3}{|c|}{ Household income status } \\
\hline Lowest & 1 & 1 \\
\hline Middle-lowest & $1.436(1.076-1.916)$ & $1.063(0.801-1.410)$ \\
\hline Middle-highest & $1.472(1.084-2.000)$ & $1.105(0.829-1.474)$ \\
\hline Highest & 1.799 (1.296-2.497) & $1.325(0.983-1.786)$ \\
\hline \multicolumn{3}{|c|}{ Occupational status } \\
\hline Manual worker & 1 & 1 \\
\hline Office worker & $1.431(1.077-1.902)$ & $1.783(1.256-2.532)$ \\
\hline Other & $0.960(0.732-1.257)$ & $1.008(0.816-1.246)$ \\
\hline \multicolumn{3}{|c|}{ Educational status (y) } \\
\hline$<6$ & 1 & 1 \\
\hline $6-8$ & $1.190(0.880-1.609)$ & $1.221(0.922-1.617)$ \\
\hline $9-11$ & $1.195(0.902-1.582)$ & $1.004(0.757-1.332)$ \\
\hline$\geq 12$ & $1.488(1.078-2.054)$ & $1.008(0.696-1.461)$ \\
\hline
\end{tabular}

ORs and $95 \%$ Cls were calculated using weighted multivariate logistic regression analyses after adjusting for age, body mass index, alcohol use, smoking, regular physical activity, daily calorie intake, average monthly household income, residence, type of health insurance, occupation, educational status, marital status, chronic disease, and cancer diagnosis.

$\mathrm{OR}$, odds ratio; $\mathrm{Cl}$, confidence interval. 
adherence to overall health check-ups after adjusting for age, body mass index, alcohol use, smoking, physical activity, daily calorie intake, average monthly household income, residence, type of health insurance, occupation, education, marital status, chronic disease, and cancer diagnosis. Compared with the lowest household income group, the ORs (95\% CIs) for adherence to overall health check-ups of the highest household income group were 1.799 (1.296-2.497) in men and 1.325 (0.983-1.786) in women. Compared with manual workers, the ORs (95\% CIs) of office workers were significant in both sexes: 1.431 (1.077$1.902)$ in men and $1.783(1.256-2.532)$ in women. In addition, the ORs
(95\% CIs) of the most educated group ( $\geq 12$ years) compared with the least educated group ( $<6$ years) were significant in men, but not in women: 1.488 (1.078-2.054) and 1.008 (0.696-1.461), respectively.

To further investigate the association between socioeconomic status and adherence to health check-ups, logistic regression analyses were performed after stratifying opportunistic and national health checkups according to socioeconomic status (Tables 3, 4). Compared with the lowest household income group, ORs (95\% CIs) for adherence to opportunistic health check-ups of the highest household income group were significantly higher (2.380 [1.218-4.653] in men and 4.042

Table 3. Adherence to opportunistic health check-ups according to socioeconomic status

\begin{tabular}{|c|c|c|c|c|c|c|}
\hline \multirow{3}{*}{ Variable } & \multicolumn{6}{|c|}{ aOR $(95 \% \mathrm{Cl})$} \\
\hline & \multicolumn{3}{|c|}{ Men } & \multicolumn{3}{|c|}{ Women } \\
\hline & Model 1 & Model 2 & Model 3 & Model 1 & Model 2 & Model 3 \\
\hline \multicolumn{7}{|c|}{ Household income status } \\
\hline Lowest & 1 & 1 & 1 & 1 & 1 & 1 \\
\hline Middle-lowest & $1.328(0.798-2.208)$ & $1.796(0.942-3.422)$ & $1.796(0.942-3.422)$ & $1.271(0.868-1.860)$ & $2.633(1.411-4.915)$ & $2.598(1.400-4.820)$ \\
\hline Middle-highest & $1.469(0.857-2.516)$ & 1.739 (0.889-3.402) & $1.738(0.887-3.408)$ & $1.478(0.992-2.200)$ & $2.865(1.587-5.171)$ & $2.827(1.567-5.099)$ \\
\hline Highest & $1.903(1.103-3.282)$ & $2.383(1.224-4.640)$ & $2.380(1.218-4.653)$ & $2.317(1.585-3.386)$ & $4.118(2.274-7.458)$ & $4.042(2.239-7.297)$ \\
\hline \multicolumn{7}{|c|}{ Occupational status } \\
\hline Manual worker & 1 & 1 & 1 & 1 & 1 & 1 \\
\hline Office worker & 1.457 (1.010-2.103) & $1.393(0.893-2.174)$ & $1.393(0.894-2.170)$ & $1.514(0.910-2.518)$ & $1.424(0.766-2.646)$ & $1.426(0.762-2.666)$ \\
\hline Other & $0.697(0.464-1.047)$ & $0.740(0.452-1.214)$ & $0.739(0.450-1.213)$ & $1.480(1.092-2.007)$ & $1.450(0.984-2.136)$ & 1.419 (1.960-2.098) \\
\hline \multicolumn{7}{|c|}{ Educational status (y) } \\
\hline$<6$ & 1 & 1 & 1 & 1 & 1 & 1 \\
\hline $6-8$ & $1.270(0.767-2.101)$ & $1.341(0.726-2.475)$ & $1.340(0.726-2.474)$ & $1.694(1.106-2.595)$ & $1.583(0.875-2.866)$ & $1.561(0.861-2.832)$ \\
\hline $9-11$ & $1.691(1.093-2.616)$ & $1.690(0.971-2.943)$ & $1.690(0.970-2.944)$ & $1.828(1.207-2.767)$ & $1.886(1.038-3.427)$ & $1.849(1.016-3.363)$ \\
\hline$\geq 12$ & $1.829(1.106-3.023)$ & $2.120(1.144-3.930)$ & $2.121(1.142-3.936)$ & $1.970(1.197-3.241)$ & $2.477(1.289-4.762)$ & $2.475(1.283-4.775)$ \\
\hline
\end{tabular}

aORs and $95 \%$ Cls were calculated using weighted multivariate logistic regression analyses after adjusting for the following variables: in model 1, age, average monthly household income, residence, type of health insurance, occupation, educational status, and marital status; in model 2, body mass index, daily calorie intake, smoking, alcohol use, and physical activity in addition to the variables in model 1; and in model 3 , chronic disease and cancer diagnosis in addition to the variables in model 2. aOR, adjusted odds ratio; $\mathrm{Cl}$, confidence interval.

Table 4. Adherence to national health check-ups according to socioeconomic status

\begin{tabular}{|c|c|c|c|c|c|c|}
\hline \multirow{3}{*}{ Variable } & \multicolumn{6}{|c|}{ aOR (95\% Cl) } \\
\hline & \multicolumn{3}{|c|}{ Men } & \multicolumn{3}{|c|}{ Women } \\
\hline & Model 1 & Model 2 & Model 3 & Model 1 & Model 2 & Model 3 \\
\hline \multicolumn{7}{|c|}{ Household income status } \\
\hline Lowest & 1 & 1 & 1 & 1 & 1 & 1 \\
\hline Middle-lowest & 1.231 (0.999-1.517) & $1.280(0.974-1.681)$ & 1.289 (0.979-1.697) & $0.985(0.824-1.177)$ & $0.988(0.750-1.301)$ & $0.988(0.751-1.300)$ \\
\hline Middle-highest & 1.501 (1.201-1.877) & $1.359(1.021-1.809)$ & 1.359 (1.019-1.812) & $1.152(0.945-1.405)$ & $1.097(0.828-1.454)$ & $1.098(0.828-1.456)$ \\
\hline Highest & $1.492(1.177-1.892)$ & $1.456(1.066-1.988)$ & 1.446 (1.059-1.974) & 1.057 (0.882-1.266) & $1.009(0.765-1.330)$ & $1.018(0.772-1.343)$ \\
\hline \multicolumn{7}{|c|}{ Occupational status } \\
\hline Manual worker & 1 & 1 & 1 & 1 & 1 & 1 \\
\hline Office worker & $1.113(0.901-1.374)$ & $1.208(0.936-1.559)$ & 1.205 (0.934-1.555) & $1.386(1.066-1.801)$ & 1.527 (1.096-2.127) & 1.508 (1.083-2.101) \\
\hline Other & $0.841(0.680-1.040)$ & $0.966(0.740-1.261)$ & $0.963(0.738-1.256)$ & $0.817(0.709-0.942)$ & $0.932(0.768-1.132)$ & $0.932(0.767-1.133)$ \\
\hline \multicolumn{7}{|c|}{ Educational status (y) } \\
\hline$<6$ & 1 & 1 & 1 & 1 & 1 & 1 \\
\hline $6-8$ & 1.123 (0.878-1.438) & 1.197 (0.891-1.607) & 1.196 (0.889-1.609) & $1.090(0.901-1.318)$ & $1.175(0.897-1.539)$ & $1.186(0.905-1.554)$ \\
\hline $9-11$ & 1.056 (0.857-1.301) & $1.024(0.793-1.322)$ & $1.031(0.798-1.332)$ & 0.985 (0.820-1.183) & $0.942(0.714-1.243)$ & $0.954(0.723-1.260)$ \\
\hline$\geq 12$ & $1.222(0.940-1.589)$ & $1.220(0.885-1.680)$ & 1.238 (0.899-1.705) & $0.746(0.585-0.950)$ & $0.714(0.501-1.017)$ & $0.725(0.508-1.034)$ \\
\hline
\end{tabular}

aORs and 95\% Cls were calculated using weighted multivariate logistic regression analyses after adjusting for the following variables: in model 1 , age, average monthly household income, residence, type of health insurance, occupation, educational status, and marital status; in model 2, body mass index, daily calorie intake, smoking, alcohol use, and physical activity in addition to the variables in model 1; and in model 3, chronic disease and cancer diagnosis in addition to the variables in model 2. aOR, adjusted odds ratio; $\mathrm{Cl}$, confidence interval. 
[2.239-7.297] in women) (Table 3), whereas those for adherence to national health check-ups in the highest household income group were also higher (1.446 [1.059-1.974] in men, but not in women 1.018 [0.772-1.343]) in model 3 (Table 4). Compared with manual workers, the ORs (95\% CIs) for adherence to opportunistic health check-ups for office workers were $1.393(0.894-2.170)$ in men and 1.426 (0.762-2.666) in women (Table 3), whereas those for adherence to national health check-ups were 1.205 (0.934-1.555) in men and 1.508 (1.083-2.101) in women after being fully adjusted (Table 4$)$. Furthermore, compared with the least educated group ( $<6$ years), the ORs for adherence to opportunistic health check-ups in the most educated group ( $\geq 12$ years) were significant (2.121 [1.142-3.936] in men and 2.475 [1.283-4.775] in women) in model 3 (Table 3 ). Regarding national health check-up performance, the ORs in the most educated group ( $\geq 12$ years) were 1.238 (0.899-1.705) for men and 0.725 (0.508-1.034) for women compared with the least educated group (<6 years) in model 3 (Table 4 ).

\section{DISCUSSION}

The major findings in this study were that individuals of both sexes with a higher socioeconomic status were more likely to undergo health check-ups. These findings were derived from a nationally representative sample. In particular, men with a higher income appear more likely to undergo opportunistic and national health check-ups, and women with a higher income are more likely to undergo opportunistic health check-ups. Female office workers appeared to undergo national health check-ups at a higher rate than female manual workers. Furthermore, more educated men underwent more opportunistic health check-ups and more educated women underwent more opportunistic and national health check-ups. These findings are consistent with previous studies. For instance, a Korean report indicated disparities in breast, cervical, and gastric cancer screening in low socioeconomic status groups. ${ }^{15,16)}$ Moreover, an Austrian study showed that a higher income and higher education level are predictors of adherence to health check-ups in men and women. ${ }^{17)}$ A European study reported that these inequalities are more prominent in countries without population-based screening programs than they are in countries with such programs. $^{18)}$

Noncommunicable diseases, which are lifestyle-related diseases, could be prevented by behavior modification and early detection, and therefore, screening tests are vital not only for early detection of noncommunicable diseases but also as a cost-effective measure from a public health perspective. ${ }^{1)}$ In South Korea, there are several types of national health check-ups, programmed by age, that are provided by the National Health Insurance Corporation. General health check-ups are provided for all people over 40 years of age, including blood tests for hemoglobin levels, fasting glucose levels, lipid profile, and liver and kidney function tests; oral examinations; chest radiography; and dementia screening tests every 2 years. Cancer screening programs for five major cancers are provided nationally, including stomach cancer for all individuals aged over 40 years, liver cancer for individuals with hepatitis B or C or liver cirrhosis, colorectal cancer for individuals over 50 years, breast cancer for women over 40 years, and uterine cervical cancer for women over 30 years. The following screening methods are performed: for stomach cancer, biennial gastric endoscopy or upper gastrointestinal series; for liver cancer, annual ultrasound and serum $\alpha$-fetoprotein test; for colorectal cancer, annual fecal occult blood test; for breast cancer, biennial mammography with palpation; and for uterine cervical cancer, a biennial pap smear test. ${ }^{2)}$ Furthermore, many private hospitals and health screening centers provide opportunistic cancer screening or health check-up programs that are easily accessible. In general, individuals willing to pay can use these programs, and they can choose the range of the screening tests.

Socioeconomic status may influence healthcare utilization in various ways. Individuals with a higher socioeconomic status have the financial leeway to easily gain access to qualified hospitals and health maintenance systems, whereas individuals with a lower socioeconomic status may have difficulty gaining access to the healthcare system due to economic barriers, informational disparity, lack of perception of need, weak support from their employer to use the healthcare system (time, expense, and transportation), and fear of additional expenses. ${ }^{9-11)}$ There are several distorted stereotypes of national health check-up programs, which might be stronger in low socioeconomic status groups. For example, "national" may be perceived as more untrustworthy than "opportunistic" programs, or the "national health check-up program" may be considered as being not for healthy people, but for people with symptoms. Such misperceptions are a barrier for low socioeconomic status groups make use of national health checkups. In addition, socioeconomic status is related to health behaviors such as physical activity, alcohol consumption, cigarette smoking, nutrition, and treatment compliance, ${ }^{3-5,7)}$ and many studies have demonstrated the association between socioeconomic status and disease outcomes and mortality. ${ }^{19,20)}$ It has been reported that potential cancer deaths can be reduced by over $30 \%$ by eliminating educational and racial disparities in the United States. ${ }^{7)}$ Korean studies have shown that education, occupation, and income level are associated with disease mortality. ${ }^{21)}$ Therefore, health inequality is an important social, ethical, and health issue both worldwide and in South Korea. ${ }^{22-24)}$ Encouraging health check-ups among individuals with a low socioeconomic status is a good first step to decreasing health inequality.

In the present study, the higher the income level, the higher the adherence rate of national screening in men, but there was no statistically significant difference in women. Moreover, women, but not men, with a higher education level tend to participate in national health checkups. Generally, individuals with a higher socioeconomic status are more likely to recognize the meaning of national screening, but the reason for the different results in men and women was not clear. A report from the Ministry of Gender Equality and Family suggested the gap may be explained by the greater difficulty women have to make time to participate in health check-ups due to their multidisciplinary role as a mother, wife, and worker. ${ }^{25)}$ Further studies are needed to elucidate the compliance of national health screening according to sex 
and its related factors.

The Korean government set up Health Plan 2010 and National Health Plan 2020 aimed toward achieving health equity. However, these remain in a diagnostic stage of exploring and measuring what the problems are. ${ }^{26)}$ Understanding the mechanism by which individuals with a low socioeconomic status experience difficulty using health check-up programs and subsequent active interventions with evidence-based policy-making and implementation are needed to reduce health inequality. ${ }^{26)}$ First, efforts to increase physical and economic access to health check-up programs among individuals with a low socioeconomic status must be given political and systemic consideration. Second, providing education and accurate information regarding national health check-ups to individuals with a low socioeconomic status is encouraged. Third, quality control for the health checkup programs and the referral system for those who have a newly diagnosed disease to be referred to primary healthcare centers will create a strong foundation for the post-diagnostic process of health check-ups, as well as improve satisfaction and trust among participants. Fourth, diversity cases from foreign countries, such as the WISEWOMEN project in the United States and the National Health Service Health Check Program (Putting Prevention First) in the United Kingdom, can be references for resolving health inequality issues. ${ }^{27,28)}$

This study has several limitations that should be considered when interpreting the findings. First, this was a cross-sectional study, and thus we could not confirm whether low socioeconomic status caused low health check-up participation. Second, we could not fully exclude the effect of reporting bias, as we used data from self-reported questionnaires. As results of self-reported surveys regarding anticipated answers sorted by socially undesirable groups tend to be underestimated owing to social desirability factors, individuals that actually should have been classified as having a lower socioeconomic status might have been classified as having a higher socioeconomic status. Third, screening participation can be affected by various factors such as personal, familial, social, organizational, and cultural factors. In addition, we cannot exclude the possibility that occupational health check-ups or industrial screenings required at certain times might have had some influence on this study. ${ }^{29)}$ There are many hidden confounding factors that we could not consider. Fourth, our analysis combined the people who have national health insurance and those who use medical aid. Subgroup analysis or comparisons between these two groups would be helpful to assess compliance with respect to health check-ups.

Despite these limitations, to the best of our knowledge, this is the first study to examine the features of the association between socioeconomic status and adherence to opportunistic and national health check-ups in Korea. Furthermore, there are several strengths in our study. First, we applied sampling weights to all analyses to retain representativeness to the entire national Korean population while controlling for possible confounding variables. Second, health check-ups were reclassified into opportunistic and national check-ups. Third, screening participation data were limited to the last 2 years in order to investigate whether socioeconomic status was associated with recent adherence to health check-ups.

In conclusion, a higher socioeconomic status was associated with a higher participation rate in health check-ups. More efforts to identify the factors associated with disparity in adherence to health check-ups are needed. Furthermore, government interventions are vital to continue quality control of health check-up programs and follow-up measures not only for health promotion but also for equality in the distribution of limited health resources. ${ }^{2,30}$

\section{CONFLICT OF INTEREST}

No potential conflict of interest relevant to this article was reported.

\section{ACKNOWLEDGMENTS}

We appreciate the members working at the Korea Centers for Disease Control and Prevention and individuals participating in the KNHANES V.

\section{SUPPLEMENTARY MATERIALS}

Supplementary materials can be found via https://doi.org/10.4082/ kjfm.2018.39.2.114. Supplementary Figure 1. Study population.

\section{REFERENCES}

1. Yach D, Hawkes C, Gould CL, Hofman KJ. The global burden of chronic diseases: overcoming impediments to prevention and control. JAMA 2004;291:2616-22.

2. Cho B, Lee CM. Current situation of national health screening systems in Korea. J Korean Med Assoc 2011;54:666-9.

3. Khang YH, Cho HJ. Socioeconomic inequality in cigarette smoking: trends by gender, age, and socioeconomic position in South Korea, 1989-2003. Prev Med 2006;42:415-22.

4. Ford ES, Merritt RK, Heath GW, Powell KE, Washburn RA, Kriska A, et al. Physical activity behaviors in lower and higher socioeconomic status populations. Am J Epidemiol 1991;133:1246-56.

5. Kontis V, Mathers CD, Rehm J, Stevens GA, Shield KD, Bonita R, et al. Contribution of six risk factors to achieving the $25 \times 25$ non-communicable disease mortality reduction target: a modelling study. Lancet 2014;384:427-37.

6. Zhao Y, You J, Wright J, Guthridge SL, Lee AH. Health inequity in the Northern Territory, Australia. Int J Equity Health 2013;12:79.

7. Siegel R, Ward E, Brawley O, Jemal A. Cancer statistics, 2011: the impact of eliminating socioeconomic and racial disparities on premature cancer deaths. CA Cancer J Clin 2011;61:212-36.

8. Yoon YS, Oh SW, Park HS. Socioeconomic status in relation to obesity and abdominal obesity in Korean adults: a focus on sex differences. Obesity (Silver Spring) 2006;14:909-19.

9. Dunlop S, Coyte PC, McIsaac W. Socio-economic status and the utilisation of physicians' services: results from the Canadian National Population Health Survey. Soc Sci Med 2000;51:123-33. 
10. Lee YY, Jun JK, Suh M, Park BY, Kim Y, Choi KS. Barriers to cancer screening among medical aid program recipients in the Republic of Korea: a qualitative study. Asian Pac J Cancer Prev 2014;15:589-94.

11. Lee SH, Joh HK, Kim S, Oh SW, Lee CM, Kwon H. Income disparities in the use of health screening services among university students in Korea: a cross-sectional study of 2479 participants in a university. Medicine (Baltimore) 2016;95:e3681.

12. Shin HY, Kang HT. Recent trends in the prevalence of chronic kidney disease in Korean adults: Korean National Health and Nutrition Examination Survey from 1998 to 2013. J Nephrol 2016;29:799-807.

13. American Heart Association. American Heart Association recommendation for physical activity in adults [Internet]. Dallas (TX): American Heart Association; 2014 [cited 2017 Jan 30]. Available from: http://www. heart.org/HEARTORG/HealthyLiving/PhysicalActivity/FitnessBasics/ American-Heart-Association-Recommendations-for-Physical-Activityin-Adults_UCM_307976_Article.jsp\#.WInAJNKLSUk.

14. The Korean Nutrition Society. Nutritional assessment program, 'Canpro 3.0' [CD-ROM]. Seoul: The Korean Nutrition Society; 2006.

15. Park MJ, Park EC, Choi KS, Jun JK, Lee HY. Sociodemographic gradients in breast and cervical cancer screening in Korea: the Korean National Cancer Screening Survey (KNCSS) 2005-2009. BMC Cancer 2011;11:257.

16. Lee M, Park EC, Chang HS, Kwon JA, Yoo KB, Kim TH. Socioeconomic disparity in cervical cancer screening among Korean women: 19982010. BMC Public Health 2013;13:553.

17. Brunner-Ziegler S, Rieder A, Stein KV, Koppensteiner R, Hoffmann K, Dorner TE. Predictors of participation in preventive health examinations in Austria. BMC Public Health 2013;13:1138.

18. Palencia L, Espelt A, Rodriguez-Sanz M, Puigpinos R, Pons-Vigues M, Pasarin MI, et al. Socio-economic inequalities in breast and cervical cancer screening practices in Europe: influence of the type of screening program. Int J Epidemiol 2010;39:757-65.

19. Khang YH, Kim HR. Relationship of education, occupation, and income with mortality in a representative longitudinal study of South Korea. Eur J Epidemiol 2005;20:217-20.

20. Khang YH, Kim HR. Explaining socioeconomic inequality in mortality among South Koreans: an examination of multiple pathways in a nationally representative longitudinal study. Int J Epidemiol 2005;34:6307.

21. Khang YH, Lynch JW, Kaplan GA. Health inequalities in Korea: ageand sex-specific educational differences in the 10 leading causes of death. Int J Epidemiol 2004;33:299-308.

22. Kim YM, Kim MH. Health inequalities in Korea: current conditions and implications. J Prev Med Public Health 2007;40:431-8.

23. Closing the gap in a generation: health equity through action on the social determinants of health. Proceedings of an international conference based on the work of the Commission on Social Determinants of Health, 6-7 November 2008, London, UK. Glob Health Promot 2009;Suppl 1:1-118.

24. Mackenbach JP, Bakker MJ; European Network on Interventions and Policies to Reduce Inequalities in Health. Tackling socioeconomic inequalities in health: analysis of European experiences. Lancet 2003;362:1409-14.

25. Kim YT, Jang MH, Kim DS, Song CS, Jang SR, Kim SM. Gender impact assessment on the national screening health tasks: centering on general health screening task, health screening task at life transitional time, health screening task for cancer, and health screening task for youth. Seoul: Ministry of Gender Equality and Family; 2011.

26. Khang YH, Lee SI. Health inequalities policy in Korea: current status and future challenges. J Korean Med Sci 2012;27 Suppl:S33-40.

27. Nahhas GJ, Daguise V, Ortaglia A, Merchant AT. Determinants of major cardiovascular risk factors among participants of the South Carolina WISEWOMAN program, 2009-2012. Prev Chronic Dis 2014;11:E153.

28. Abdalrahman B, Soljak M. NHS health checks: an update on the debate and program implementation in England. J Ambul Care Manage 2015;38:5-9.

29. Gochman DS. Health behavior research: definition and diversity. In: Gochman DS, editor. Handbook of health behavior research I: personal and social determinants. New York (NY): Plenum Press; 1997. p. 3-20.

30. Kim YS, Lee JA. National health examination expansion policy. J Korean Med Assoc 2017;60:104-7. 


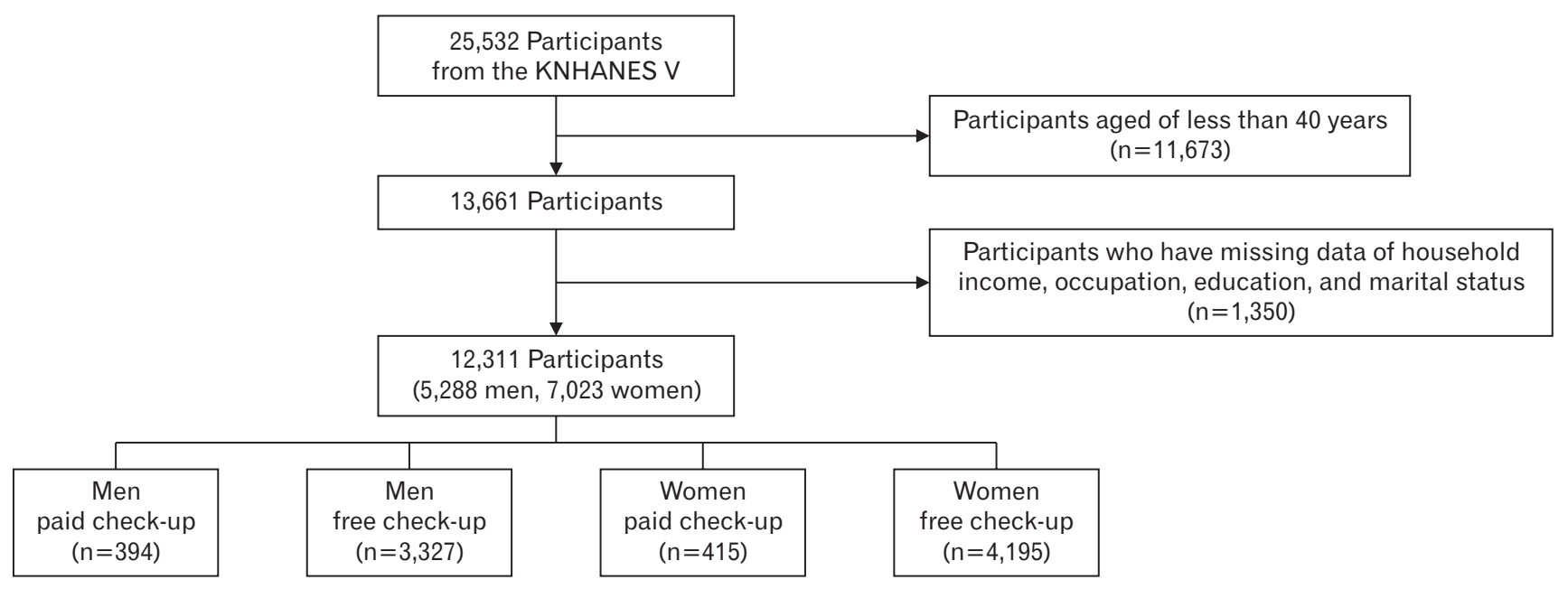

Supplementary Figure 1. Study population of the present study. KNHANES, Korean National Health and Nutrition Examination Survey. 